
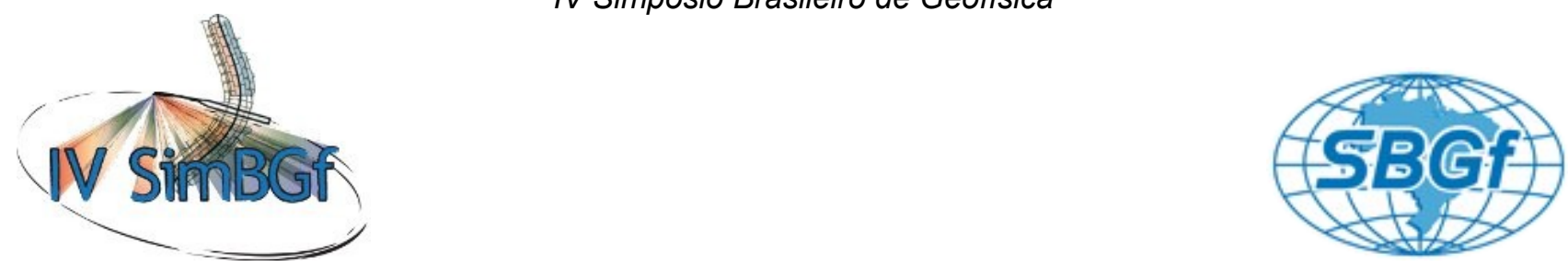

\title{
Monitoramento da atividade sísmica no Nordeste do Brasil: o exemplo da estação PFBR
}

Dantas, R.R.S. ${ }^{1,2 *}$, Ferreira, J.M. ${ }^{1,2}$, Nunes, B.I.C. ${ }^{1}$, do Nascimento, A.F. ${ }^{1}$, Moreira, M.A. ${ }^{1,2}$, Lima Neto, H.C. ${ }^{1}$, Menezes, E.A.S. ${ }^{1}$

1. Departamento de Geofísica, CCET / UFRN

2. Projeto RSISNE (Petrobras/UFRN/FUNPEC)

Copyright 2010, SBGf - Sociedade Brasileira de Geofísica

Este texto foi preparado para a apresentação no IV Simpósio Brasileiro de Geofísica, Brasilia, 14 a 17 de novembro de 2010. Seu conteúdo foi revisado pelo Comitê Técnico do IV SimBGf, mas não necessariamente representa a opinião da SBGf ou de seus associados. É proibida a reprodução total ou parcial deste material para propósitos comerciais sem prévia autorização da SBGf.

\section{Resumo}

A atividade sísmica do Nordeste é uma das maiores do país. Visando monitorar e conhecer mais aprofundadamente a sismicidade da região, cinco estações foram instaladas pela UFRN, com recursos do Projeto do Milênio. Uma dessas estações é a de Pau dos Ferros (PFBR), localizada no Oeste Potiguar. Usando os dados coletados pela estação de janeiro de 2009 até março de 2010, medindo-se os tempos de chegada das ondas $\mathrm{P}$ e $\mathrm{S}$ e o azimute em relação à estação de sismos locais e regionais, e fazendo aproximações a partir dos modelo de velocidades já conhecido da Província Borborema, estimou-se a localização de alguns epicentros, principalmente localizados nas áreas sísmicas de Tabuleiro Grande e da barragem do Castanhão, mostrando a potencialidade de monitoramento de uma única estação.

\section{Introdução}

O Nordeste é a região de maior sismicidade no Brasil, e, por esse motivo, há vários projetos focados no estudo da atividade sísmica nessa região. Em 2007, dentro do Projeto do Milênio, a UFRN instalou 5 estações sismográficas do tipo banda larga triaxiais nos estados do Rio Grande do Norte, Paraíba, Pernambuco e Ceará. Hoje, essa rede continua em operação sendo que três dessas estações foram realocadas. As estações inicialmente instaladas foram: Pau dos Ferros (RN), Sobral (CE), Ocara (CE), Solânea (PB) e Agrestina (PE) (Figura 1). A função principal dessas estações é monitorar a atividade sísmica na Província Borborema, Nordeste Brasileiro. A partir de meados de 2010, uma rede permanente RSISNE (Petrobras), com 15 estações, será instalada na região (da Bahia ao Piauí) para o monitoramento da atividade sísmica. Todas essas estações são providas de sensores triaxiais de banda larga e registradores digitais. Como parte do treinamento na análise dos dados da rede RSISNE, os dados da rede do Milênio foram sistematicamente analisados a partir de janeiro de 2009. O objetivo deste trabalho é mostrar os resultados desse monitoramento, a partir dos registros da estação PFBR, instalada no município de Pau dos Ferros (RN). Suas coordenadas são $-6.12^{\circ} \mathrm{E},-38.27 \mathrm{~W}$ e altitude de $219.6 \mathrm{~m}$, assim, localizada próximo aos limites dos estados do Rio Grande do Norte e Ceará, que está dentro da região sismogênica conhecida como borda da Bacia Potiguar, a mais ativa do país.

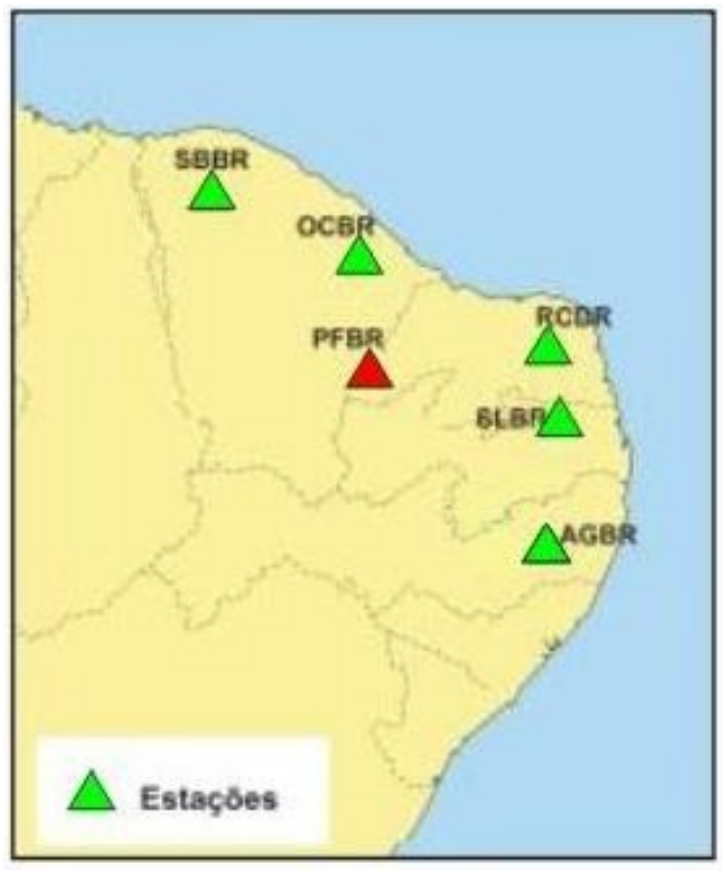

Figura 1: Localização das estações do Projeto Milênio.

\section{Metodologia}

Os movimentos do solo, detectados por sensor com resposta em velocidade, foram registrados pelo sismógrafo, armazenados em seu disco rígido e coletados por visitas periódicas à estação. Esses dados foram analisados, no laboratório, com o auxílio do software Dimas. Essa análise consistiu, primeiramente, em identificar sismos nos arquivos contínuos de uma hora de duração cada, com taxa de 50 amostragens por segundo, e utilizando o filtro IIR Butherworth Bandpass 5$20 \mathrm{~Hz}$. Os sismos foram analisados tendo por objetivo a determinação do epicentro utilizando o registro triaxial dos mesmos. Os parâmetros que foram obtidos são 
distância epicentral e azimute, em relação à estação. Para a determinação da distância epicentral $(\Delta)$ foram lidos os tempos de chegada das ondas $P$ e $S\left(t_{P}\right.$ e $\left.t_{S}\right)$ (Figura 2). Como a distância epicentral é muito maior que a profundidade do hipocentro, e usando um modelo regional em que a ondas percorrem uma camada semiinfinita homogênea em trajetória retilínea do hipocentro até a estação, podemos aproximar a distância epicentral a partir da equação

$$
\Delta=k \cdot\left(t_{S}-t_{P}\right)
$$

em que k é uma constante obtida a partir do modelo regional de velocidades como sendo $8.7 \mathrm{~m} / \mathrm{s}$. Sendo $v_{p} a$ velocidade das ondas $P$ e $v_{s}$ a velocidade das ondas $S$, tem-se que:

$k=\frac{v_{P} \cdot v_{S}}{v_{P}-v_{S}}$

O azimute foi obtido a partir da análise do primeiro movimento de partícula, utilizando o software Dimas (Figura 3). Tanto o azimute como a distância epicentral são usadas para se estimar a localização do epicentro, plotando em mapa o ponto. A partir das coordenadas estimadas dos epicentros, um mapa foi gerado com a ajuda do software livre QGIS (plataforma Linux), utilizando alguns sismos selecionados (Figuras 4 e 5).

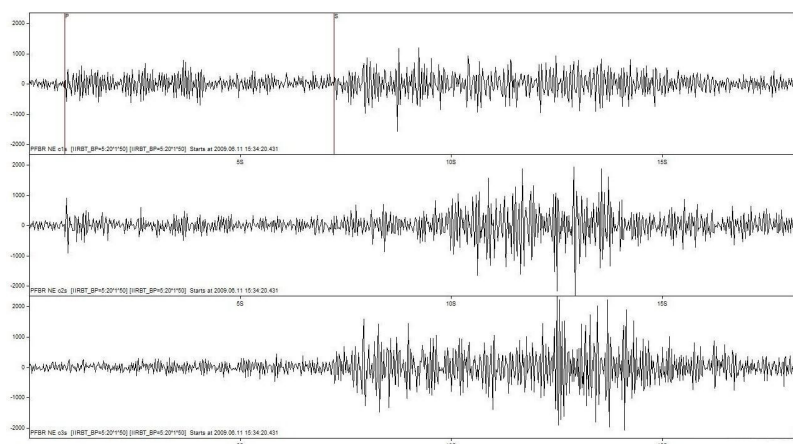

Figura 2: Sismograma de um evento registrado em PFBR, em ambiente Dimas. Os tempos de chegada das ondas $P$ e $S$ estão marcados na primeira componente (vertical).

\section{Resultados}

Com a análise de cada evento encontrado nos sismogramas, gerou-se uma lista de eventos registrados pela estação, a partir de janeiro de 2009. Foram registrados mais de 500 eventos, incluindo sismos e explosões. No entanto, para eventos regionais $(\Delta>100$ $\mathrm{km})$, nem sempre foi possível determinar o azimute. Para sismos locais $(\Delta<100 \mathrm{~km})$ as principais áreas sísmicas ativas durante o período em tela foram a de Tabuleiro Grande (RN) e da área de inundação do açude do Castanhão (CE). Registros de sismos regionais importantes como de Taipu (RN; 4.3 Mb), Alagoinha (PE), Santana do Matos (RN), entre outros, foram analisados e suas leituras contribuíram para a determinação do
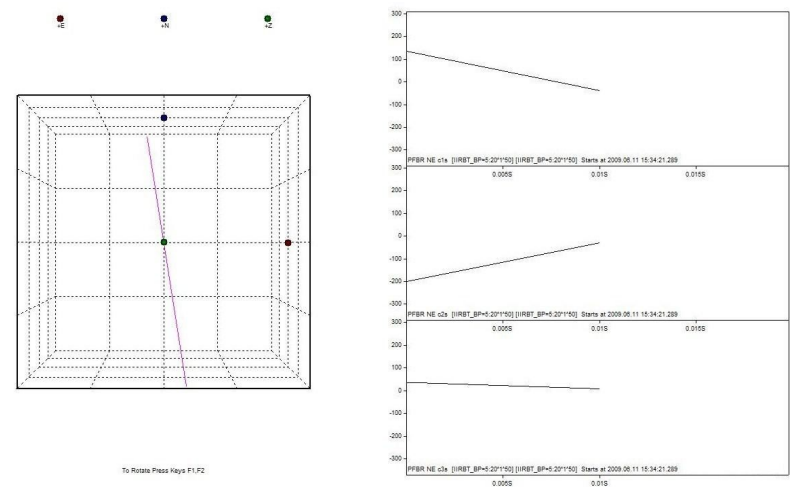

Figura 3: Primeiro movimento de partícula do sismograma acima, em ambiente Dimas.

epicentro e magnitude. Além desses resultados, esse alto índice de eventos oriundos da área a noroeste da estação PFBR mostrou que, mesmo distante, a estação possibilita um monitoramento dos maiores eventos ocorridos na área sismogênica do Castanhão. Nessa região, no momento, está instalada uma rede local para estudos de detalhe, composta por seis estações, a saber: CSMI, CSMY (Mycaela), CSGB (Gado Bravo), CSSF (São Francisco), CSBF (Belas Flores) e CSFS (Fazenda da Serra). A partir dos dados coletados por essa rede no período compreendido entre os dias 21/11/2009 e 14/03/2010, construiu-se um mapa epicentral da área cercada pela rede, usando o software livre GMT (plataforma Linux) (Figura 6).

\section{Discussão e Conclusões}

Por sua localização, a estação PFBR consegue monitorar ampla área da Província Borborema, principalmente a borda da Bacia Potiguar. Seus dados foram e continuarão sendo importantes para um melhor conhecimento da sismicidade da região. Os resultados obtidos, com uma só estação, demonstram a potencialidade que terá uma rede permanente de 15 estações para o monitoramento da atividade sísmica no Nordeste do Brasil. Essa estação também registrou eventos que começaram e continuam a incentivar pesquisadores da área a detalhar seus estudos nas regiões sismogênicas que foram identificadas pela análise dos eventos registrados pela estação.

\section{Agradecimentos}

Ao Projeto do Milênio e ao INCT de Estudos Tectônicos, pela disponibilização dos dados.

Ao Projeto RSISNE (Petrobras/UFRN/FUNPEC) pelo estágio.

\section{Referências}

FERREIRA, J.M., OLIVEIRA, R.T., TAKEYA, M.K. \& ASSUMPÇÃO, M., 1998. Superposition of local and regional stresses in northeast Brazil: evidence from focal mechanisms around the Potiguar marginal basin. Geophys. J. Int., 134: 341-355. 
NUNES, B.I.C., 2010. Estudo da atividade sísmica na área do reservatório do açude do Castanhão. Tese de Doutorado. Em elaboração.

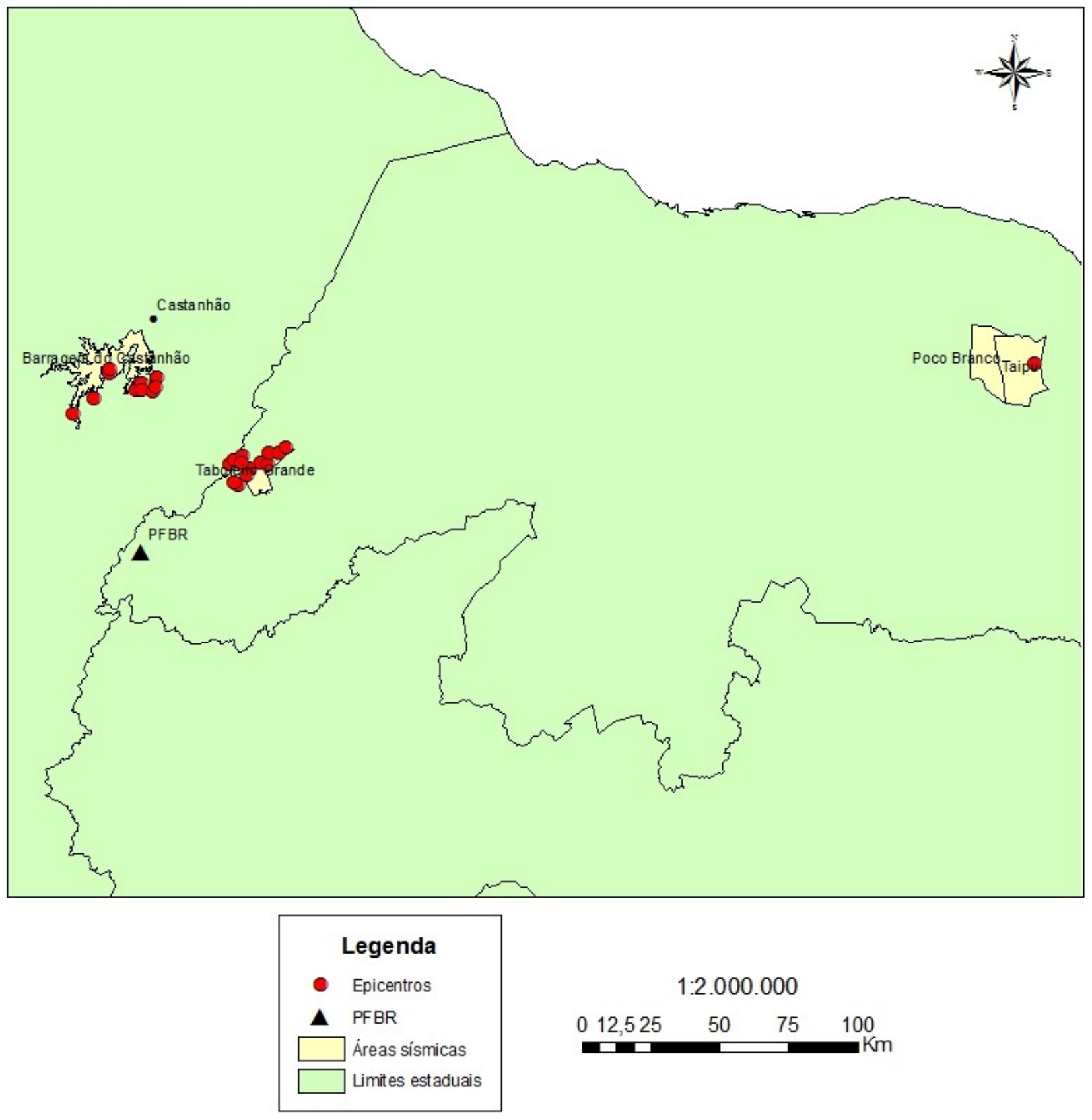

Figura 4: Mapa de localização epicentral da porção setentrional do Nordeste brasileiro, com eventos registrados pela estação PFBR, com destaque para as áreas de Taipu, Tabuleiro Grande e da barragem do Castanhão. 


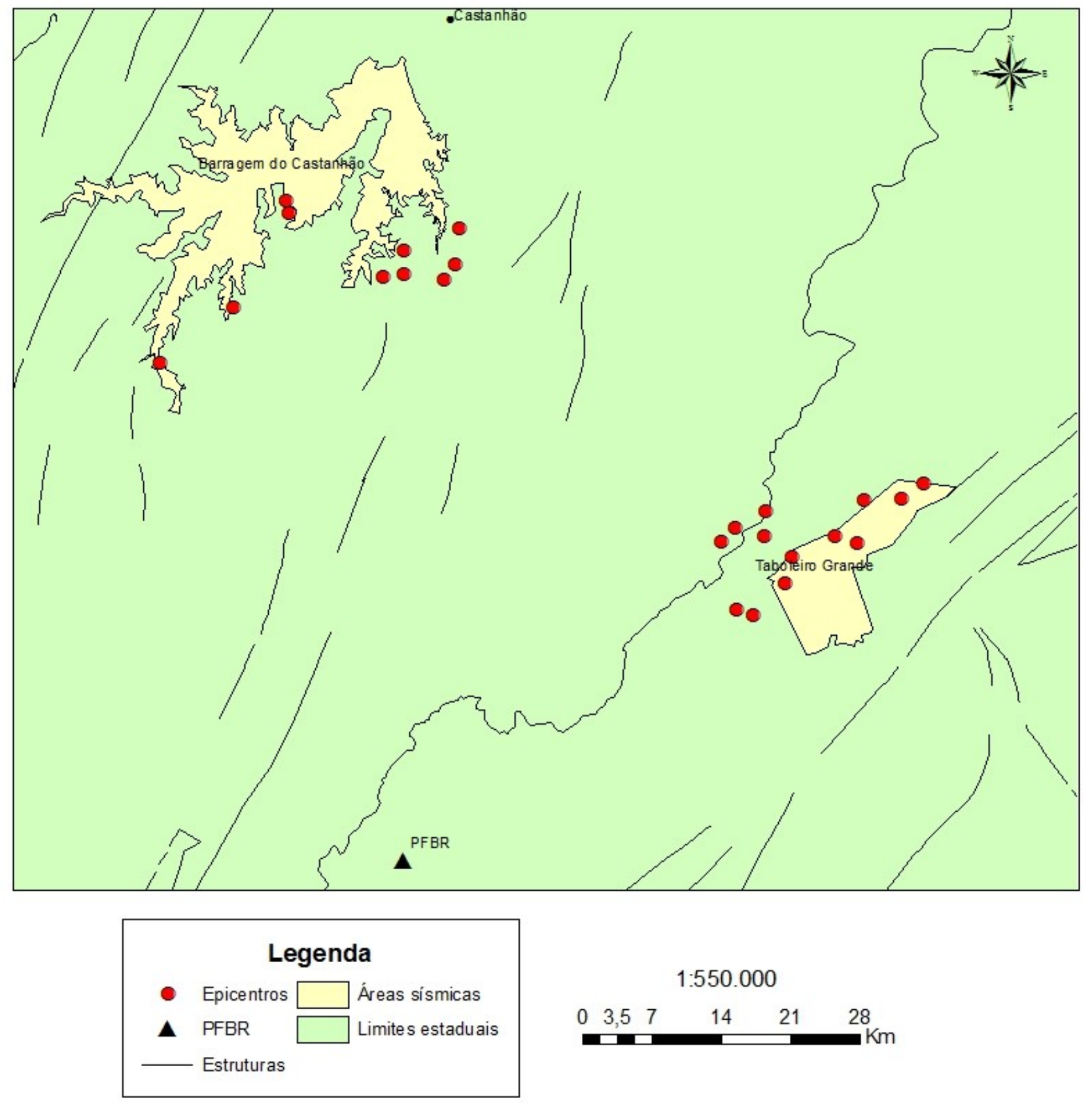

Figura 5: Mapa de localização epicentral local de eventos selecionados registrados pela estação PFBR, com estruturas regionais e destaque para as áreas de Tabuleiro Grande e da barragem do Castanhão. 


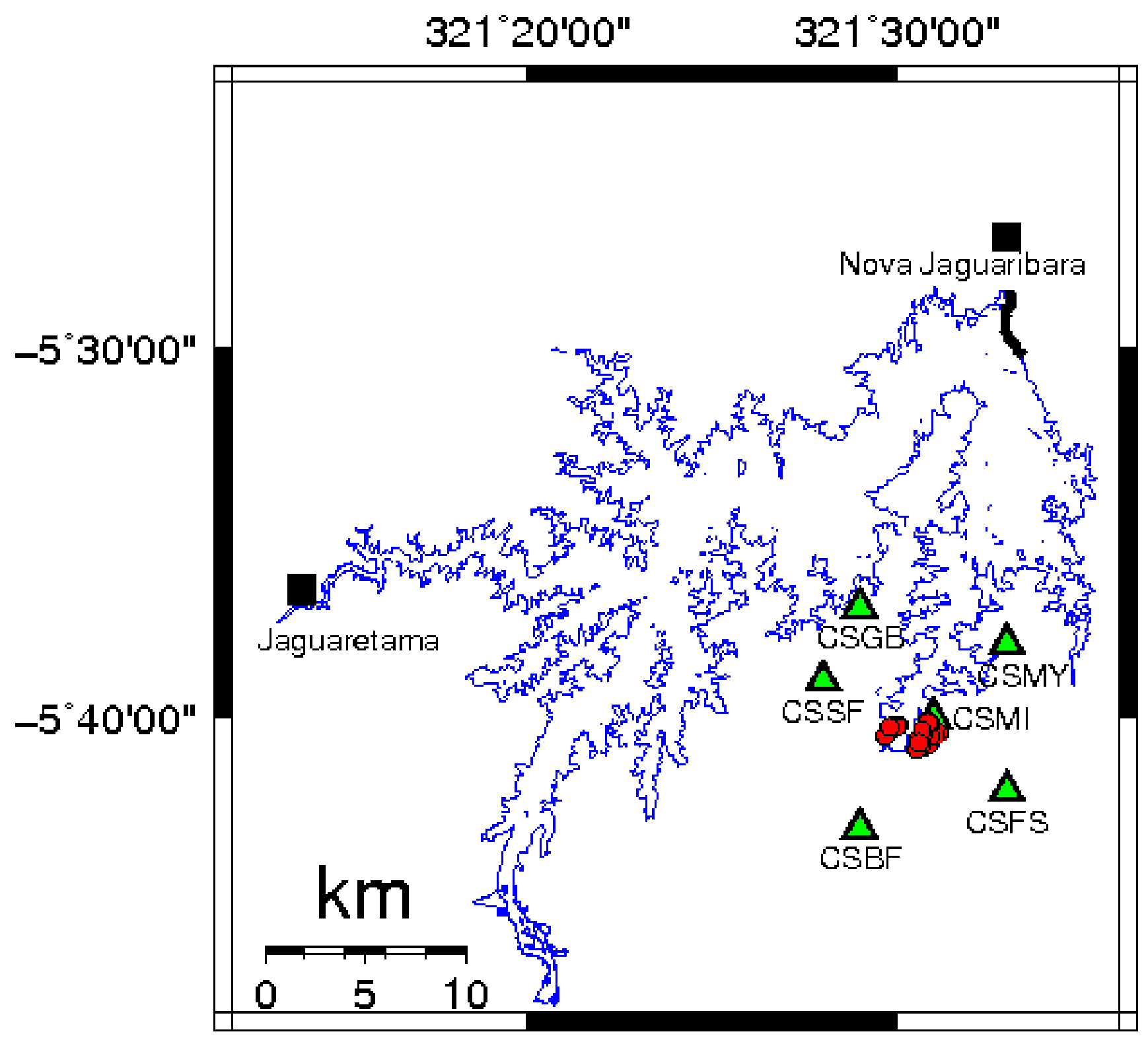

Figura 6: Mapa de localização epicentral de eventos registrados pela rede local instalada para monitorar a atividade sísmica da barragem do Castanhão. 